\title{
Direct measurement of the energy dissipated by quantum turbulence
}

\author{
D. I. Bradley ${ }^{\star}$, S. N. Fisher, A. M. Guénault, R. P. Haley, G. R. Pickett, D. Potts and V. Tsepelin
}

The lack of a general solution to the governing Navier-Stokes equations means that there is no fundamental theory of turbulence. In the simpler case of pure quantum turbulence, the tangle of identical singly quantized vortices in superfluids at $\boldsymbol{T} \sim \mathbf{0}$ may provide a deeper understanding of turbulence in general. The well-known Kolmogorov theory ${ }^{1}$ predicts the energy distribution of turbulence and how it decays. In normal systems the turbulent energy is generally only a small perturbation on the total thermal energy of the supporting medium. In quantum turbulence, however, the energy is accessible. A stationary condensate is necessarily in its ground state with zero enthalpy. Thus quantum turbulence accounts for the entire free energy of the superfluid and there are no other contributions. Here, we exploit this property to make the first direct measurement of the energy released by freely decaying quantum turbulence. Our results are consistent with a Kolmogorov energy spectrum with an inferred Kolmogorov constant remarkably similar to those of classical fluids.

The turbulent energy is an important quantity here. Vortex lines contribute two energy components to the system (see Fig. 1), the loss of condensation energy in the vortex core, where the superfluidity is suppressed (negligible in our case), and the kinetic energy in the flow fields surrounding the cores. In earlier experiments on quantum turbulence the quantity usually inferred from experiment is the average vortex-line density $L$ (the vortexline length per unit volume $)^{2}$. Comparison with the velocityfield characterization of classical turbulence usually relies on a phenomenological model ${ }^{3}$ equating the line density to the mean square vorticity of classical turbulence: $\left\langle\omega^{2}\right\rangle=\kappa^{2} L^{2}$, with $\kappa$ the circulation quantum. An effective kinematic viscosity $v^{\prime}$ is also introduced, enabling the dissipation per unit mass to be written $\epsilon=v^{\prime} \kappa^{2} L^{2}$ in analogy with the classical turbulence dissipation $\epsilon=v\left\langle\omega^{2}\right\rangle$, where $v$ is the classical kinematic viscosity ${ }^{2,3}$ (the true viscosity of a pure superfluid being identically zero). Although this model has not been directly tested, it agrees qualitatively with measurements of the vortex line density $L(t)$ for decaying quantum turbulence ${ }^{4}$ in superfluid ${ }^{3} \mathrm{He}-\mathrm{B}$ and in superfluid ${ }^{4} \mathrm{He}$ (refs 3,5), allowing a determination of $v^{\prime}$. In contrast, a direct measurement of the energy dissipated in quantum turbulence allows us to test the standard model of decaying turbulence independently of this phenomenological model.

In the experiment presented here, the energy dissipation is measured directly with a 'black-body radiator' (BBR; ref. 6) the main features of which are shown in Fig. 2. The BBR is a thin-walled box with a small orifice in one side, immersed in superfluid ${ }^{3} \mathrm{He}-\mathrm{B}$, and cooled by nuclear refrigeration. Inside the radiator is a thermometer wire resonator, a heater wire resonator to inject power, and a very low-amplitude resonating grid, which generates the turbulence by the initial production of microscopic vortex rings, as discussed in the Methods section. The grid resonator consists of

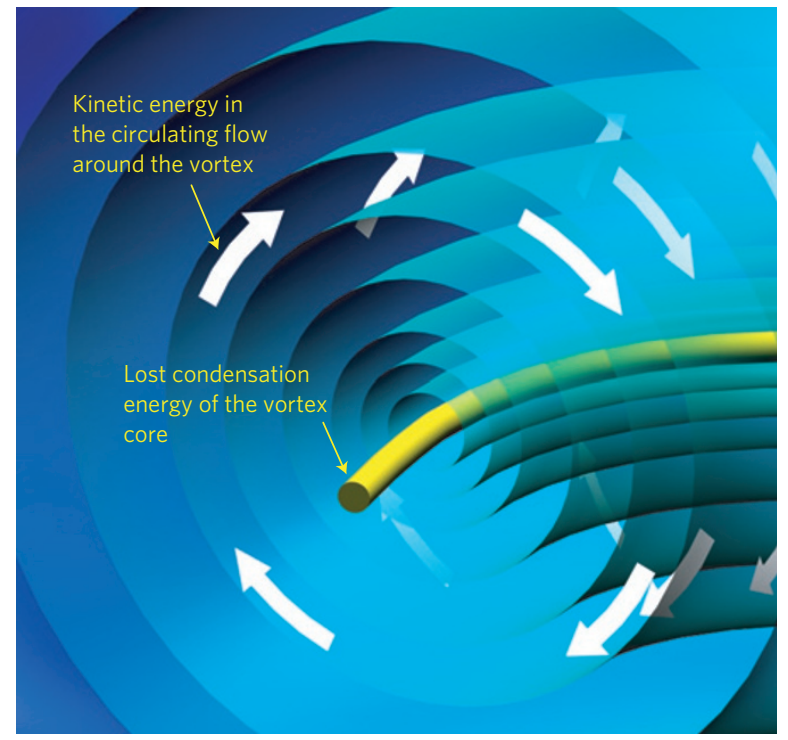

Figure 1 | The energy distribution in pure quantum turbulence. At the $T=0$ limit, a coherent condensate carrying turbulence has only two contributions to the energy; first, the loss of condensation energy in the vortex core (in yellow) arising from the suppression of the superfluidity along the linear singularity of the vortex, and second, the kinetic energy of the surrounding flow circulating the core, as indicated by the arrows.

a $5 \times 5 \mathrm{~mm}$ goalpost shaped Ta wire carrying a $5 \times 3.5 \mathrm{~mm} \mathrm{Cu}$ grid mesh of $\sim 197$ lines $\mathrm{cm}^{-1}$.

The experiments are performed in the low-temperature ballistic regime below $200 \mu \mathrm{K}$, where the thermal quasiparticle excitation mean free path is greater than the container dimension. (All data presented here were taken at a pressure of $3.3 \mathrm{bar}$, where the superfluid transition temperature, $T_{\mathrm{C}}$, is $1.32 \mathrm{mK}$.) Heat entering the radiator from any source produces ballistic quasiparticles, which thermalize by scattering off the walls, finally emerging as a beam of excitations from the orifice. At steady state, the power emitted in the beam balances the power entering the radiator. From simple kinetic theory, the power emitted ${ }^{6}$ is given by $\dot{Q}_{\text {beam }}=\left\langle n v_{\mathrm{g}}\right\rangle \tilde{E}_{T} A / 4$, where $\left\langle n v_{\mathrm{g}}\right\rangle$ is the thermal flux of excitations of mean energy $\tilde{E}_{T}=\Delta+k_{\mathrm{B}} T$, $\Delta$ is the superfluid energy gap, and $A$ the effective area of the orifice. The damping on the thermometer wire is dominated by quasiparticle scattering, which being well understood ${ }^{7}$ provides very sensitive thermometry ${ }^{8}$. The thermal flux of excitations can therefore be inferred from the measured damping on the thermometer wire ${ }^{9}$, and from this quantity we obtain the power leaving the BBR (as described in the Methods section below). When turbulence is present, the excess power leaving the BBR provides a direct measure of the energy being released by the freely decaying 


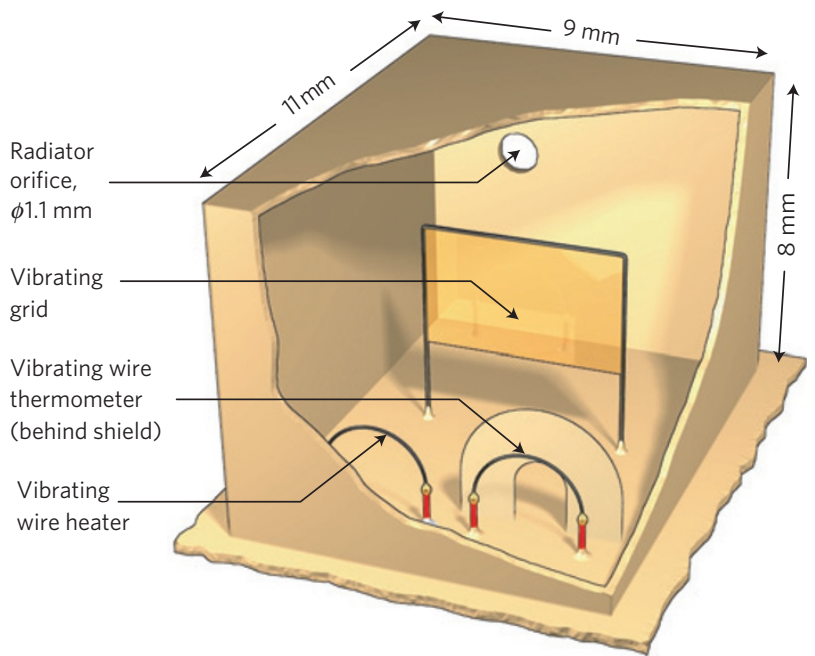

Figure 2 | The quasiparticle black-body radiator. The BBR is indeed a black-body radiator, but for quasiparticle excitations rather than conventionally for photons. Inside the 'box', one vibrating-wire resonator acts as a thermometer by probing the quasiparticle excitation flux. A second acts as a heater by injecting power directly into the superfluid when accelerated beyond the critical velocity for pair breaking. The grid oscillates at $\sim 1 \mathrm{kHz}$, but with negligible amplitude (of order hundreds of nanometres), to create the turbulence. The box acts a bolometer measuring the energy released by the decaying turbulence inside the enclosure.

turbulence, and this allows us to reconstruct the energy content of the original turbulence.

Figure 3 shows the excess power leaving the BBR as a function of time after switching off the drive to the grid. This data was taken for an initial grid velocity of $4.51 \mathrm{~mm} \mathrm{~s}^{-1}$. While driven, the grid generates both vortices and large numbers of quasiparticle excitations ${ }^{10}$. In the absence of vortices, we expect the excess power to decay exponentially with the BBR time constant, $\delta \dot{Q}=\dot{Q}_{0} \exp \left(-t / \tau_{i}\right)$. This is estimated from simple kinetic theory as $\tau_{i} \simeq 4 V /\left(\left\langle v_{\mathrm{g}}\right\rangle A\right)$, with $V$ the volume of the BBR and $\left\langle v_{\mathrm{g}}\right\rangle$ the mean excitation group velocity ${ }^{8}$. The BBR dimensions were designed to give a time constant of order $\tau_{i} \sim 0.5 \mathrm{~s}$. A series of similar measurements were made for a range of initial grid velocities. All the data presented here were taken at 3.3 bar.

The green lines in the figure show the initial exponential recovery with an intrinsic time constant of $\tau_{i}=0.54 \mathrm{~s}$, in good agreement with the design value. At later times the excess power decays much more slowly, revealing a much longer-lived source of excitations in the BBR. This is the dissipation of the quantum turbulence produced by the grid. We can subtract the initial thermal recovery, $\delta \dot{Q}=\dot{Q}_{0} \exp (-t / \tau)$, to leave only the contribution from the slowly decaying vortices. This is shown in Fig. 4, where we plot the dissipation from the turbulence as a function of time for various initial grid velocities. We note that the thermal recovery is only significant during the first few seconds.

From earlier measurements ${ }^{11}$, confirmed by computer simulations $^{12}$, we know that at low grid velocities the generated vortex rings travel ballistically. The rings have a self-propagation speed $^{13}$ of around $\sim 10 \mathrm{~mm} \mathrm{~s}^{-1}$, corresponding to diameters of $\sim 5 \mu \mathrm{m}$. In the current experiment, such rings would collide with the BBR walls after $\sim 0.5 \mathrm{~s}$. As we expect these rings to dissipate rapidly after colliding with a wall, they will not contribute to the later-time dissipation shown in Fig. 4. Above some critical grid velocity the ring density becomes sufficient for collisions, and reconnecting rings then rapidly evolve into quantum turbulence ${ }^{11,12}$. The critical velocity for this grid is found to be $\sim 2 \mathrm{~mm} \mathrm{~s}^{-1}$, because there is no measurable turbulence dissipation below this velocity.

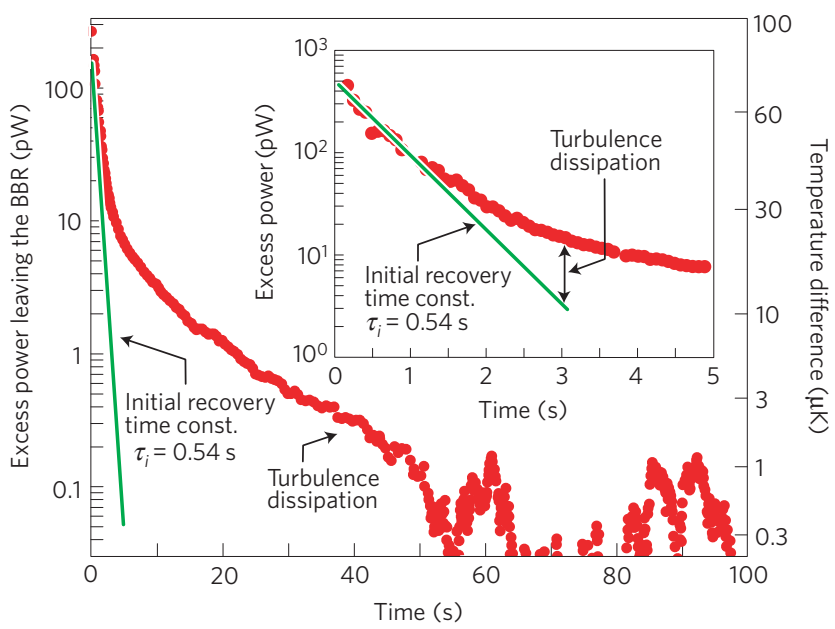

Figure 3 | The excess power leaving the BBR as a function of time after stopping the grid. While the grid is oscillating to produce turbulence, simultaneous pair breaking also produces a large excess of quasiparticle excitations. These are rapidly emitted through the orifice into the bulk superfluid outside, at a rate governed by the BBR time constant $\tau$ (see text). The measured early-time behaviour is shown inset, marking an exponential recovery with time constant close to the design value for the BBR, as shown by the solid lines. Thus the temperature in the box falls to its very low, equilibrium temperature in a few seconds. Further heat emission then represents the decay of the quantum turbulence in the condensate, which is now cold again, as shown by the late-time behaviour in the main figure. The initial grid velocity was $4.5 \mathrm{~mm} \mathrm{~s}^{-1}$. The right-hand scale shows the equivalent temperature difference between the liquid in the BBR and the bulk.

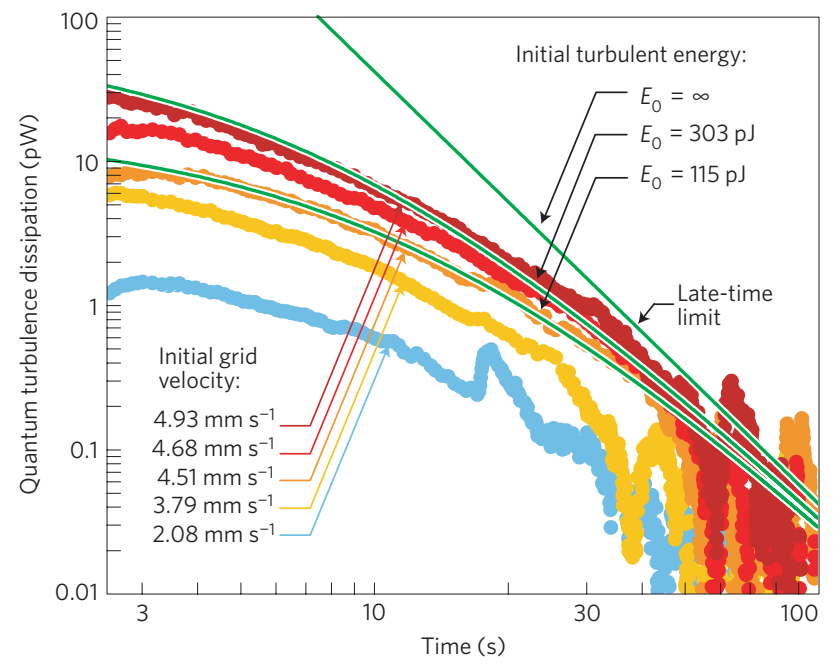

Figure 4 | The power dissipated by decaying quantum turbulence produced by the grid. The figure shows the power dissipated for various initial grid velocities. (Below an initial grid velocity of $\sim 2 \mathrm{~mm} \mathrm{~s}^{-1}$ there is essentially no turbulence signal seen above the noise, and we take this as the critical velocity for turbulence production.) The solid green lines show the expected behaviour for classical homogeneous isotropic turbulence having a Kolmogorov spectrum with a Kolmogorov constant $C$ of 2.0 .

We now compare the results with expectations from classical turbulence. According to the standard model ${ }^{1,2}$, the Richardson cascade transfers energy from large to small length scales (low to high wavenumbers, $k$ ) over an inertial range where viscosity plays no role. The energy is mostly contained in large eddies of length scale $l_{\mathrm{e}}$, but is dissipated by viscous forces at the smallest scales. 
The energy per unit wavenumber per unit mass in the inertial range is predicted by the Kolmogorov spectrum $E_{\mathrm{k}}=C \epsilon^{2 / 3} k^{-5 / 3}$, with $C$ a constant of order unity. Assuming that this spectrum spans the full range of length scales from the cell size $\left(l_{\mathrm{e}}=d \simeq 10 \mathrm{~mm}\right)$ to the smallest (dissipative) scale $a$, the total turbulent energy is obtained by integrating $E=\rho V \int_{k_{d}}^{k_{a}} E_{\mathrm{k}} \mathrm{d} k$, where $V$ is the volume of the radiator, $k_{d}=2 \pi / d$ and $k_{a}=2 \pi / a$. Assuming $a \ll d$, the dissipation $\dot{Q}=\rho V \epsilon$ is independent of the dissipative scale $a$ and given by:

$$
\dot{Q}=\rho V \frac{d^{2}}{\pi^{2}} \frac{27}{4} C^{3}\left(t_{0}+t\right)^{-3} \quad t_{0}=\frac{d}{\pi} \sqrt{\frac{27 C^{3}}{8} \frac{\rho V}{E_{0}}}
$$

where $E_{0}$ is the initial turbulent energy.

Thus the predicted late-time decay of the dissipation should be proportional to $t^{-3}$, and independent of all fluid properties other than the density. Thus the only unknown parameter is the Kolmogorov constant $C$. Therefore a measurement of the dissipated energy gives a particularly direct test of this model.

The behaviour at earlier times depends also on the initial energy $E_{0}$, which determines $t_{0}$ and introduces a second free parameter. The solid lines in Fig. 4 show the behaviour predicted by equation (1) with a Kolmogorov constant of $C=2.0$. The data at higher grid velocities are seen to agree very well with the model, with initial energies of order $100 \mathrm{pJ}$. The Kolmogorov constant extracted from the fits, $C \simeq 2.0 \pm 0.4$, falls within the range of typical values attributed to classical fluids ${ }^{14}$. The behaviour differs at the lower velocities ( $3.79 \mathrm{~mm} \mathrm{~s}^{-1}$ and below), where the late-time behaviour is consistent with a $t^{-2}$ dependence, as expected for a random tangle with no large scale structure ${ }^{2}$. (Here the rings have reconnected to form a tangle, but at too low a density to develop a Kolmogorov spectrum). A similar crossover from a random tangle to classicallike turbulence has also been inferred from measurements of the line density in both superfluid ${ }^{3} \mathrm{He}-\mathrm{B}$ and superfluid ${ }^{4} \mathrm{He}$ at low temperatures ${ }^{4,5,15}$.

Our data at high grid velocities agree well with the standard model of classical turbulence. However, there are caveats. First, in practice the initial energy spectrum will depend on the turbulenceproduction process. To explain towed-grid measurements in superfluid ${ }^{4} \mathrm{He}$, a more elaborate model $^{3}$ has been developed incorporating an initial $k^{2}$ energy spectrum at large length scales and a time-dependent energy-containing length scale $l_{\mathrm{e}}(t)$ that grows until it saturates at the container size $d$, after which the behaviour follows equation (1). This model does not yield substantially improved fits to our data, suggesting that the initial conditions do not critically influence the late-time behaviour. Second, the initial turbulence is clearly not isotropic or homogeneous ${ }^{16}$. Nevertheless, we observe very similar behaviour with wire-generated turbulence, even though the initial turbulence distributions must differ substantially, which suggests that the initial homogeneity does not affect the later-time behaviour. Third, one should question whether the vortex-line density in the BBR is large enough to provide an inertial range sufficient to support a Kolmogorov spectrum, but we note that measurements of the line density in other experiments ${ }^{4,17}$ suggest that there are no large deviations from the simple model at very low densities, even when the spatial extent $d$ corresponds to just a few line spacings. Finally, as the initial temperature in the BBR, whilst the turbulence is being generated, is quite high $\left(\sim 0.25 T_{\mathrm{C}}\right.$ or $330 \mu \mathrm{K}$ for the highest velocity data), mutual friction may influence the production process and the initial behaviour ${ }^{18}$. Fortunately, this effect rapidly becomes negligible, as the radiator promptly cools in the first few seconds.

\section{Methods}

The mechanism for generating quantum turbulence. From earlier work ${ }^{11}$, we know that when we oscillate an object in the superfluid, the flow fields lead initially to the generation of microscopic vortex rings. This process gives rise to a cloud of similar-sized rings leaving the moving object, with the rate of creation increasing with the amplitude of the oscillatory flow. Under our conditions, the rings are a few micrometres in diameter and have velocities of $\sim 10 \mathrm{~mm} \mathrm{~s}^{-1}$, which is fast on the scale of the BBR dimensions. The subsequent history of this gas of rings depends on the density. At low densities the rings travel independently and rapidly leave the generation site. At some critical density, however, the ring density is high enough that they collide and reconnect to form larger, slower structures, trapping more rings, and turbulence rapidly develops.

The generation process by the grid is thus very different from that in a classical towed-grid scenario. In the present case, the grid is essentially stationary, as the amplitude of motion at the highest grid velocities is only a few hundreds of nanometres, thus generating no significant large-scale motion in the liquid. The specific geometry of the grid essentially plays no role, as the grid is only acting as a coarse surface for assisting the generation of the gas of rings. It is perhaps better to think of this device more as a stationary planar transducer (emitting vortex rings instead of sound) rather than as a moving grid as used in classical flow experiments. Nevertheless, this flat transducer provides us with the closest we can currently get to homogeneous quantum turbulence.

Operation and calibration of the black-body radiator. The thermal damping on the thermometer wire ${ }^{9}$ is given by $\Delta f_{2}^{T}=B \exp \left(-\Delta / k_{\mathrm{B}} T\right)$. At our temperatures $(\sim 200 \mu \mathrm{K}), \Delta \gg k_{\mathrm{B}} T$, the exponential is changing very rapidly, providing an extremely precise value of $T$ (at $170 \mu \mathrm{K}$ a change of only $1 \mu \mathrm{K}$ gives rise to a change in the damping of nearly $10 \%) . \Delta f_{2}{ }^{T}$ is also proportional to $\left\langle n v_{\mathrm{g}}\right\rangle$. Thus we have the quantities needed to determine the beam power, $\dot{Q}_{\text {beam }}=\left\langle n v_{\mathrm{g}}\right\rangle \tilde{E}_{T} A / 4$. The beam power can thus be rewritten as $\dot{Q}_{\text {beam }}=c W_{T}$, where $W_{T}=\Delta f_{2}^{T} T \tilde{E}_{T}$ (which we designate the 'width parameter' of the thermometer wire), $T$ and $\tilde{E}_{T}=\Delta+k_{\mathrm{B}} T$ have been obtained directly from $\Delta f_{2}{ }^{T}$, as above, and $c$ is a calibration constant to be determined ${ }^{6}$.

On injecting large powers, the bulk superfluid outside the BBR also warms slightly, yielding an influx of excitations back through the orifice from the outside. This is accounted for by subtracting the width parameter $W^{\prime}$ measured by a similar thermometer wire located outside the BBR, close to the orifice, but shielded from the excitation beam by a paper screen. The net power leaving the radiator is thus given by ${ }^{5} \dot{Q}_{\text {out }}=c \delta W$, where $\delta W=W_{T}-W^{\prime}$. In the absence of additional sources of heating, the power leaving the radiator balances the steady-state heat leak into the BBR, $\dot{Q}_{\text {leak }}=c \delta W_{0}$.

The radiator is calibrated by measuring the increase in the width parameter $\Delta W=\delta W-\delta W_{0}$ while a measured power $\dot{Q}_{\mathrm{ap}}$ is injected into the radiator with the heater wire. A linear response is observed from which the calibration constant $c=\dot{Q}_{\mathrm{ap}} / \Delta W$ is deduced. Once calibrated, the width parameter directly measures the net power leaving the radiator. The background heat leak is found to be $\dot{Q}_{\text {leak }} \simeq 2 \mathrm{pW}$, resulting in a base temperature inside the BBR of $172 \mu \mathrm{K}\left(\sim 0.13 T_{\mathrm{C}}\right)$ whilst the surrounding superfluid cools to $\sim 145 \mu \mathrm{K}$. To determine the energy being dissipated by turbulence in the $\mathrm{BBR}$, we correct for the heat leak and refer to the excess power, leaving the BBR, $\delta \dot{Q}=\dot{Q}_{\text {out }}-\dot{Q}_{\text {leak }}$.

Received 13 December 2010; accepted 4 March 2011; published online 3 April 2011

\section{References}

1. Kolmogorov, A. N. Dissipation of energy in the locally isotropic turbulence. Dokl. Akad. Nauk. SSSR 32, 19-21 (1941) reprinted in Proc. Roy. Soc. A 434, 15-17 (1991).

2. Vinen, W. F. \& Niemela, J. J. Quantum turbulence. J. Low Temp. Phys. 128, 167-231 (2002).

3. Stalp, S. R., Skrbek, L. \& Donnelly, R. J. Decay of grid turbulence in a finite channel. Phys. Rev. Lett. 82, 4831-4834 (1999).

4. Bradley, D. I. et al. Decay of pure quantum turbulence in superfluid ${ }^{3} \mathrm{He}-\mathrm{B}$. Phys. Rev. Lett. 96, 035301 (2006).

5. Walmsley, P. M. et al. Dissipation of quantum turbulence in the zero temperature limit. Phys. Rev. Lett. 99, 265302 (2007).

6. Fisher, S. N., Guénault, A. M., Kennedy, C. J. \& Pickett, G. R. Blackbody source and detector of ballistic quasiparticles in ${ }^{3} \mathrm{He}$ B: Emission angle from a wire moving at supercritical velocity. Phys. Rev. Lett. 69, 1073-1076 (1992).

7. Enrico, M. P., Fisher, S. N. \& Watts-Tobin, R. J. Diffuse scattering model of the thermal damping of a wire moving through superfluid ${ }^{3} \mathrm{He}-\mathrm{B}$ at very low temperatures. J. Low Temp. Phys. 98, 81-89 (1995).

8. Bäuerle, C. B., Bunkov, Y. M., Fisher, S. N. \& Godfrin, H. Temperature scale and heat capacity of superfluid ${ }^{3} \mathrm{He}-\mathrm{B}$ in the $100 \mu \mathrm{K}$ range. Phys. Rev. B. 57, 14381-14386 (1998).

9. Fisher, S. N., Guénault, A. M., Kennedy, C. J. \& Pickett, G. R. Beyond the two-fluid model: Transition from linear behaviour to a velocity-independent force on a moving object in ${ }^{3}$ He. Phys. Rev. Lett. 63, 2566-2569 (1989).

10. Bradley, D. I. et al. Vortex generation in superfluid He-3 by a vibrating grid. J. Low Temp. Phys. 134, 381-386 (2004).

11. Bradley, D. I. et al. Emission of discrete vortex rings by a vibrating grid in superfluid ${ }^{3} \mathrm{He}-\mathrm{B}$ : A precursor to quantum turbulence. Phys. Rev. Lett. 95, 035302 (2005). 
12. Fujiyama, S. et al. Generation, evolution, and decay of pure quantum turbulence: A full Biot-Savart simulation. Phys. Rev. B 81, 180512 (2010).

13. Bradley, D. I. et al. Grid turbulence in superfluid ${ }^{3} \mathrm{He}-\mathrm{B}$ at low temperatures. J. Low Temp. Phys. 150, 364-372 (2008).

14. Yeung, P. K. \& and Zhou, Y. Universality of the Kolmogorov constant in numerical simulations of turbulence. Phys. Rev. E. 56, 1746-1752 (1997).

15. Walmsley, P. M. \& Golov, A. I. Quantum and quasiclassical types of superfluid turbulence. Phys. Rev. Lett. 100, 245301 (2008).

16. Bradley, D. I. et al. Fluctuations and correlations of pure quantum turbulence in superfluid ${ }^{3}$ He-B. Phys. Rev. Lett. 101, 065302 (2008)

17. Skrbek, L., Niemela, J. J. \& Donnelly, R. J. Four regimes of decaying grid turbulence in a finite channel. Phys. Rev. Lett. 85, 2973-2976 (2000).

18. Eltsov, V. B. et al. Quantum turbulence in a propagating superfluid vortex front. Phys. Rev. Lett. 99, 265301 (2007).

\section{Acknowledgements}

We acknowledge technical support from M. G. Ward and A. Stokes, and funding from the UK EPSRC, the FP7 European MICROKELVIN network and the Royal Society.

\section{Author contributions}

All the authors contributed to the devising of the experiment and the analysis of the data. The experiments were carried out by D.I.B., S.N.F. and D.P. The paper was written by D.I.B., S.N.F. and G.R.P.

\section{Additional information}

The authors declare no competing financial interests. Supplementary information accompanies this paper on www.nature.com/naturephysics. Reprints and permissions information is available online at http://npg.nature.com/reprintsandpermissions. Correspondence and requests for materials should be addressed to D.I.B. 